\title{
The Regulatory Region of Muscle-Specific Alpha Actin 1 Drives Fluorescent Protein Expression in Olive Flounder Paralichthys olivaceus
}

\author{
${ }^{\dagger}$ Hee Jeong Kong ${ }^{1}$, Julan Kim¹, Ju-Won Kim¹, Hyun-Chul Kim², Jae Koo Noh', \\ Young-Ok Kim¹, Woo-Jin Kim², Sang-Yeob Yeo ${ }^{3}$, and Jung Youn Park ${ }^{1}$ \\ ${ }^{1}$ Biotechnology Research Division, National Institute of Fisheries Science, Busan 46083, Korea \\ ${ }^{2}$ Genetics and Breeding Research Center, National Institute of Fisheries Science, Geoje 53334, Korea \\ ${ }^{3}$ Division of Applied Chemistry and Biotechnology, Hanbat National University, Daejeon 34158, Korea
}

\begin{abstract}
To develop a promoter capable of driving transgene expression in non-model fish, we identified and characterized the muscle-specific alpha-actin gene in olive flounder, Paralichthys olivaceus (PoACTC1). The regulatory region of PoACTC1 includes putative regulatory elements such as a TATA box, two MyoD binding sites, three CArG boxes, and a CCAAT box. Microinjection experiments demonstrated that the regulatory region of PoACTC1, covering from $-2,126$ bp to $+751 \mathrm{bp}$, just prior to the start codon, drove the expression of red fluorescent protein in developing zebrafish embryos and hatching olive flounder. These results suggest that the regulatory region of PoACTC1 may be useful in developing a promoter for biotechnological applications such as transgene expression in olive flounder.
\end{abstract}

Key words : Paralichthys olivaceus, Olive flounder, Alpha-actin, Microinjection, RFP, Expression

\section{INTRODUCTION}

The olive flounder, Paralichthys olivaceus, is an important marine fish species for aquaculture in Asian countries including Korea, Japan, and China (Kim et al., 2014). It is cultivated mainly on the southern coast and Jeju Island in Korea, with 37,267 tons produced in 2018, worth approximately 422 million USD, or $14.9 \%$ of the total aquaculture value in the Korea (http://kosis.kr/statHtml/statHtml. do?orgId=101\&tblId=DT_1EW0001\&conn_path=I2). Its genome and transcriptome were sequenced as a model for studying asymmetric development during flatfish metamorphosis (Shao et al., 2017).
Actins are essential cytoskeletal proteins in eukaryotic cells that play an important role in cell motility, structure, and integrity (Dominguez et al., 2011). Of the three major vertebrate actin groups, alpha-actins are specific for striated muscle, and cardiac muscle alpha actin 1 (ACTC1) is expressed in cardiac and skeletal muscle (Krasnov et al., 2003; Moll et al., 2006). The alpha-actin promoter has been a critical model for the identification of muscle-specific regulatory elements in vertebrates from fish to mammals (Sartorelli et al., 1990; Higashijima et al., 1997; Krasnov et al., 2003; Moll et al., 2006). The promoter region of the human cardiac alpha-actin (HCA) gene contains transcription factor-binding sites for MyoD, CArG

\footnotetext{
Manuscript received January 25, 2019, Received in revised form February 26, 2019, Accepted March 9, 2019

${ }^{\dagger}$ Corresponding Author : Hee Jeong Kong, Biotechnology Research Division, National Institute of Fisheries Science (NIFS), Busan 46083, Korea. Tel: +82-51-720-2457, Fax: +82-51-720-2456, E-mail: heejkong@korea.kr

This is an Open Access article distributed under the terms of the Creative Commons Attribution Non-Commercial License (http:// creative-commons.org/licenses/by-nc/3.0) which permits unrestricted non-commercial use, distribution, and reproduction in any medium, provided the original work is properly cited.
} 
box binding factor, and Sp1 upstream of the TATA box, which are required for muscle-specific expression (Gustafson \& Keds, 1989; Sartorelli et al., 1990).

The first fish alpha-actin gene was identified in the pufferfish, Fugu rubripes (Venkatesh et al., 1996). Alpha-actin promoters have been cloned and characterized in several fish species, including zebrafish Danio rerio, medaka Oryzias latipes, and channel catfish Ictalurus punctatus (Higashijima et al., 1997; Kusakabe et al., 1999; Kim et al., 2000). It is important to study an autologous regulatory region in olive flounder for basic research and biotechnological applications such as transgenesis (Beardmore, 1997; Higashijima et al., 1997).

In this study, we isolated and characterized the regulatory region of the ACTC1 gene of olive flounder P. olivaceus (PoACTC1). Pressure-controlled microinjection method was optimized for the delivery of foreign DNA into fish embryo. Its transcriptional activity was shown to direct expression of red fluorescent protein (RFP) in zebrafish and flounder embryos. This study is the first report of autologous promoter-driven transgene expression in the marine fish P. olivaceus.

\section{MATERIALS AND METHODS}

\section{Fish maintenance}

Fish were maintained at the Biotechnology Research Division of the National Institute of Fisheries Science (NIFS) in Busan, Korea. Adults (2 years old) were maintained in 1-ton fiber-reinforced plastic (FRP) tanks at an approximate density of 10 fish per tank, with a flowthrough system supplied with filtered seawater. Fish were fed a commercial fish diet (crude protein, 52\%; crude fat, $11 \%)$ three times per day. Fertilized eggs were stocked in a 100-L tank with a flow-through system supplied with filtered seawater along with ultraviolet (UV) light treatment; $98 \%$ of the eggs hatched 3 days later. The feeding program was modified from Sakakura (2006). Hatchlings were fed enriched L-type rotifers (Brachionus plicatilis complex) supplemented with chlorella twice a day starting from 3 days post hatching (dph). The temperature of the rearing tanks was maintained at $20^{\circ} \mathrm{C}$. Animal experiments were conducted in accordance with the Animal Protection Act of the Ministry of Agriculture, Food and Rural Affairs, Korea, and were approved by the NIFS (2017-NIFS-IACUC09). For microinjection into zebrafish, fish were maintained at $28.5^{\circ} \mathrm{C}$ with a 14 -h light/10-h dark cycle, as described by Westerfield (1995).

2. Cloning of POACTC1 gene from the olive flounder $P$. olivaceus

Expressed sequence tag (EST) clones were isolated from a $P$. olivaceus cDNA library using a plasmid purification kit (Intron, Seongnam, Korea), and sequenced using universal T3 reverse primer (Promega, Madison, WI, USA) on an ABI3730xl automatic sequencer (Applied Biosystems, Foster City, CA, USA). The EST clone 1before-32a-K12 showed significant sequence homology to known alpha-actin sequences. The cDNA sequence was annotated in GenBank using BLASTX (http://www.ncbi.nlm.nih.gov/ BLAST/). The PoACTCl genomic sequence was predicted by AUGUSTUS, a web server for gene prediction in eukaryotes (Stanke \& Morgenstern, 2006), using the genome sequences of $P$. olivaceus (Kim et al., unpublished data). The potential transcription factor binding sites in the PoACTCl gene using the PROMO program version 3.0 (http://alggen.lsi.upc.es/cgi-bin/promo_v3/promo/promoinit. cgi?dirDB=TF_8.3). The parameters such as "species", "factors" and "matrix" were set as "All", and "maximum matrix dissimilarity rate" was 15 .

\section{Construction of red fluorescence protein (RFP)} reporter plasmid

To create the RFP reporter plasmid for the regulatory region of PoACTC1, DNA fragments ranging from positions $-2,126$ to +751 in Po-actcl were generated by PCR 
using Vent DNA polymerase (New England BioLabs, Ipswich, MA, USA) and then inserted into the SalI and AgeI restriction sites upstream of the DsRed 2 gene in pDsRed2-1 (Clontech Laboratories, Mountain View, CA, USA). The primer sequences were as follows: PoACTC1-2126 FS, 5'-GCC GTC GAC GTT CTT TAA AGG GCT TCT ACT CTG TTT-3'; and Po-ACTC1+751RA, 5'-AAT ACC GGT GGT GTC AGC TCT GCA ACA CAC ACG GAG-3'. The construct was confirmed by sequencing.

\section{Microinjection into fish embryos}

The fluorescent protein reporter construct of the regulatory region of PoACTC1 (pPoACTC1-2877bp) was digested with the restriction endonuclease SalI (New England BioLabs). The purified DNA solution (25 $\mathrm{ng} / \mathrm{uL}$ ) was injected into one-cell-stage zebrafish embryos $(\mathrm{n}=100)$ using an air pressure microinjector (PicoPump820, World Precision Instruments, Sarasota, FL, USA), as described previously (Kong et al., 2013). For the microinjection into olive flounder, the method of preparing microinjection needle modified to that described in Goto et al. (2015). Glass microinjection needles were produced by pulling of a borosilicate glass capillary tubes (World Precision Instruments, Inc., Sarasota, FL, USA) using micropipette puller device (Narishige, Tokyo, Japan). End of the tip was melted slightly in a loop of platinum wire to form constriction preventing backflow of cytoplasm and grinded with micropipette grinder (Narishige) to permit smooth penetration into the cytoplasm. The DNA solution (25 ng/uL) was injected into two-cell-stage flounder embryos $(\mathrm{n}=100)$ (Kim et al., unpublished data). The estimated average amount of DNA delivered to each embryo was $15-20 \mathrm{pg}$. Digital images of the embryos were captured using a macro zoom fluorescence microscope (MVX10; Olympus, Tokyo, Japan). The expression rates of red fluorescence were calculated with randomly chosen embryos.

\section{RESULTS AND DISCUSSION}

1. Cloning and characteristics of the regulatory region of the Po-actc1 gene

We isolated PoACTCl cDNA from the olive flounder $P$. olivaceus by screening a flounder cDNA library (data not shown). The EST clone 1before-3-2a-K12, which carries a 1,336-bp insertion, showed significant sequence homology to known alpha-actin sequences, including a predicted flounder actin mRNA sequence (GenBank no. XM_020111 908). The PoACTCl genomic sequence $(4,117 \mathrm{bp})$ was predicted by the AUGUSTUS gene prediction software and the obtained sequence was confirmed by PCR and sequencing. The sequence contained the PoACTCl gene, covering from 2,126 bp upstream of the putative transcription start site to the fifth exon of PoACTCl cDNA, which includes the first introns.

The muscle-specific expression of the cardiac alphaactin gene requires transcription factors such as MyoD1, CArG box binding factor, and Sp1 upstream of the TATA box (Gustafson \& Keds, 1989; Sartorelli et al., 1990). We identified potential transcription factor binding sites in the PoACTCl gene using the PROMO program version 3.0. The proximal promoter region of PoACTCl contains putative regulatory elements such as a TATA box (TATAAATT) at -21 to $-14 \mathrm{bp}$, a MyoD binding site at -155 to -148 bp (ACAGATGT), and two CArG boxes at -78 to $-67 \mathrm{bp}$ (CCAAATATGGAG) and -176 to $-165 \mathrm{bp}$ (CTCCATAGATGG) from the putative transcription start site (Fig. 1). The pentameric GCTGC sequence associated with the MyoD binding site (Sartotrlli, 1990) was also found in the regulatory region of the PoACTCl gene. The proximal promoter region of the HCA gene, $-117 \mathrm{bp}$ from the start site of transcription, is sufficient to convey muscle-specific expression to heterologous reporters (Minty \& Keds, 1986).

The PoACTCl promoter also contains various transcription factor binding elements, including an additional 
A

-2126 GTTCTTTAAAGGGCTTCTACTCTGTTTATGATGTGGAATTCCTATAGAAAATCAATATGTTTTTTGTTCTGGACAGTTTTGTCCTTATTATCTCATCTCGGCTGCTGAGTAATGAGGCCG -2007 ATCTCCTGAGGGATCGTGAGCAGGAGGAGAATGATCGTGCCACAACGATTTAATCTCAGTAGCAACAGCTACAGTGAAAGTCACACTGCAGGACTTTGAGCAGACTCCAAGTGTCTCTGT

ATCTAATCCGGGCTCTGGCTCGAGTTCTGGGAGTAAGTGAAACATCTATTTTACAGTTAAACAATATTATCATGTACATCCTGAACCCTTCGTCTGACAGTGATGGTTCCTGAGCACACT

1646

1526

-1527

$-140$ CCAAT

AGGCAATGTCATTTCCCATGTCTACTTCTTCCCTCAGTGCAGGAGTAAAGAAAGAATATGGCACATGTCACAACAAGAGATGTTCCTTTTGCTTCATCGTTTGGATGTCGGCAACAATAA -1287

-1286 ATTACGTTAACATTGCTTTTGATTTATCTGTAAAGGTTTCTTACACGAAGGAATACATTACCTAGACTTTACATAAAAAGATAATCATTAATCCAGTGGCTGGTGTTTGAGGTATGAGTA -1167

-1166 TGTGTTTGGATGGAGGACAAGAAATATGCCAAGAAGAATTATATCACTGCACCAGATAACCTTTGATAATATACTTGGGTCAAAGTATTAAAGTTTCCTCCGAAGGCACCGGAAATAGTG -1047

-1046 TTAGTCTGTTATGTATGAATATTTCTGTCTGACTGGTCAATAAGAAAGTAAAATATGCAAAAGAAGAACCATGGGAGTATGAATTCTAATTAATCTGAAATATTTTCACTTGCAAACACA -927

-926 GCATGTTGCATGGCCACCGCATCAATAACTTCAAAGAAGCTCATTATAATAATGAAAACTCATTGATTTGATGTAAATCAAATATGTGAATGCATCGGCAAACATATGTTCTCATTTACC -807

B

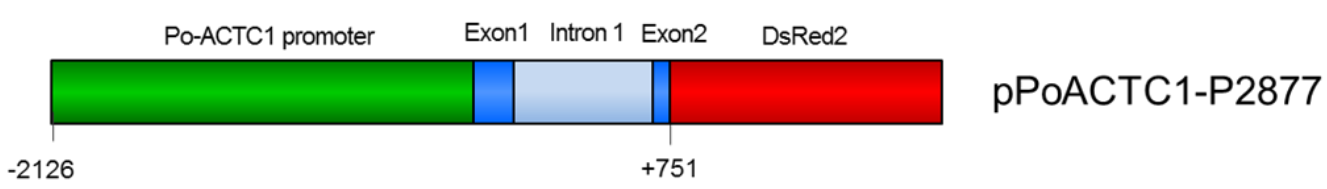

Fig. 1. Nucleotide sequences and schematic representation of the fluorescent protein reporter of the regulatory region of Po-ACTC1. (A) The exons are shown in blue. The start codon (ATG) is shown in bold and underlined. The predicted transcription factor bind-ing sites are shown in pink. (B) The gene fragment of the Po-ACTCl gene was fused with red fluores-cent protein (DsRed2) gene.

CArG-box at -804 to $-797 \mathrm{bp}$, a MyoD binding site at 644 to $-637 \mathrm{bp}$, and a CCAAT box at $-1,455$ to $-1,451 \mathrm{bp}$ in the $5^{\prime}$ upstream promoter region. Several of these elements contribute to the cooperative transcription-activating interaction of the cardiac alpha-actin gene in cellular processes for cell motility, structure, and integrity (Miwa \& Kedes, 1987; Bunnell et al., 2011). Further studies may reveal the detailed function and related mechanism of regulatory element(s) in the expression of PoACTC1.

\section{Regulatory region of Po-actc1 gene in zebrafish} and olive flounder

To examine activation of the regulatory region of $\mathrm{Po}$ $A C T C 1$, we performed a fluorescence microscopy analysis using RFP expression driven by the PoACTCl regulatory region in zebrafish and olive flounder muscle. In zebrafish, between $9.8 \%$ ( 3 out of 51 ) and $46.9 \%$ (23 out of 49 ) of the surviving embryos microinjected with pPoACTC1P2877 exhibited red fluorescence at 2 day post-fertilization (dpf) (Fig. 2A). In olive flounder, pPoACTC1-P2877 also 

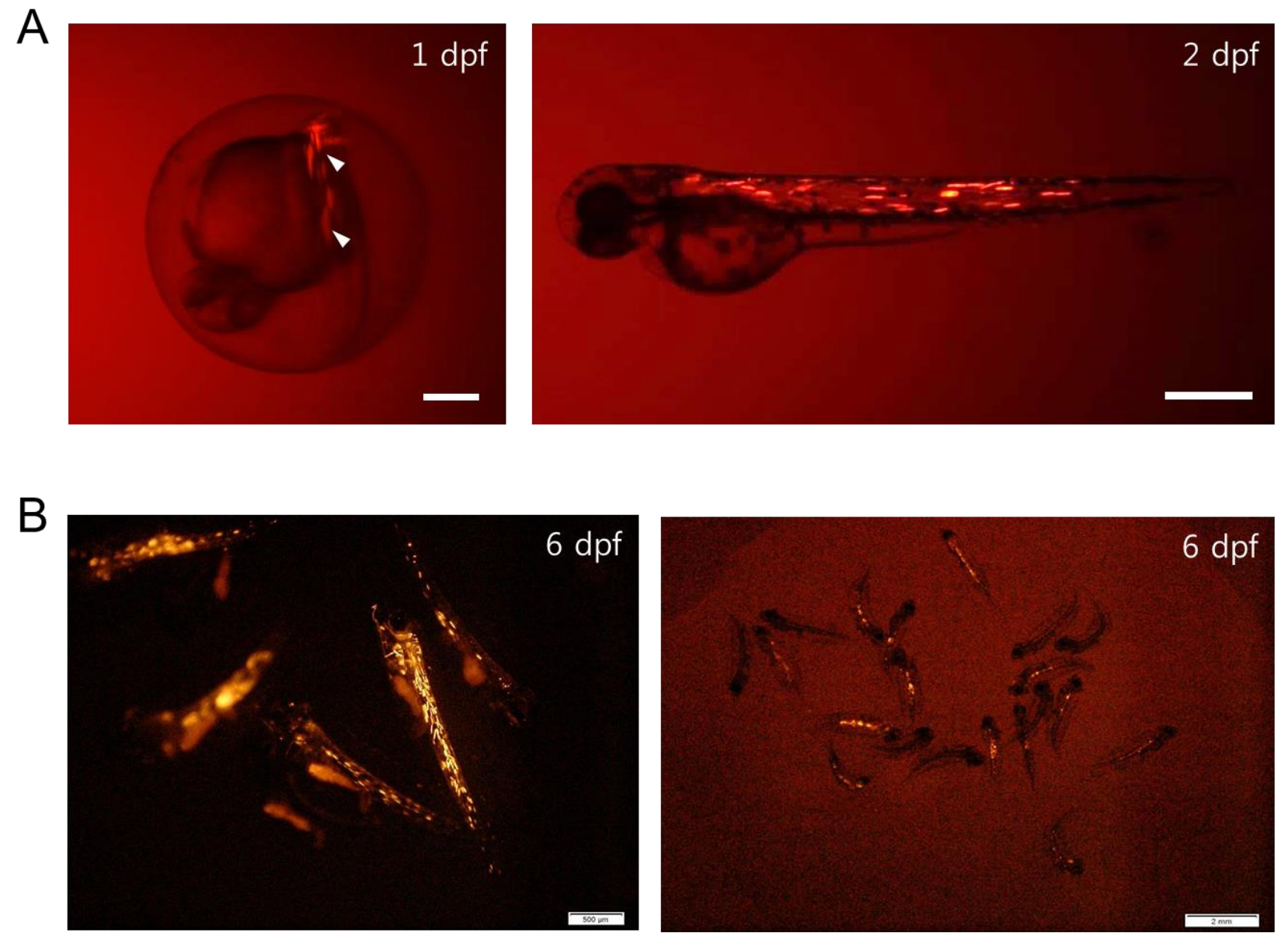

Fig. 2. Activation of the regulatory region of $P$ A ACTC1 in zebrafish embryos and olive flounder. The fluorescent protein reporter of the PoACTC1 regulatory region (pPoACTC1-P2877) was digested with the restriction endonuclease SalI, then the DNA solution was injected into one- or two-cell-stage embryos using an air pressure microinjector. Digital images of embryos were captured using an Olympus macro zoom fluorescence microscope. (A) Zebrafish embryos at 1 and $2 \mathrm{dpf}$; scale bar: $200 \mu \mathrm{m}$. (B) Olive flounder at $6 \mathrm{dpf}$; scale bar: $500 \mu \mathrm{m}$.

drove RFP expression at $2 \mathrm{dpf}$, and the signal was still detected at $6 \mathrm{dpf}$ (Fig. 2B). The expression rates of the pPoACTC1-P2877 constructs in olive flounder were between $58.5 \%$ (24 out of 41$)$ and $60 \%$ (12 out of 20$)$ at 6 dpf. Various techniques have been developed to perform gene delivery of exogenous DNA, including microinjection and electroporation. Microinjection of DNA into fish embryos began from its initial development in fertilized rainbow trout (Oncorhynchus mykiss) in the 1980s (Mclean et al., 1984). Although many studies have reported gene delivery and transgenesis in fish, from model fish such as zebrafish and medaka to aquaculture species such as Nile tilapia and common carp (Tonelli et al., 2017), this is the first report of microinjection and expression of a foreign gene driven by an autologous promoter in embryos of the marine fish olive flounder. We have modified the microinjection protocols to overcome the problem that the hard chorion of its embryo makes microinjection complicated. The success of microinjection and identification of autologous tissue-specific promoter would be influential for basic and applicative studies, which might be helpful to improve the valuable trait of olive flounder.

Here, we described the cloning and characterization of the PoACTC1 regulatory region in olive flounder $P$. oliva- 
ceus, a major aquaculture fish in Korea. We demonstrated the activation of the PoACTCl regulatory region in a fluorescence reporter assay using microinjection into zebrafish and olive flounder embryos. These results lay the groundwork for the development of a useful promoter for transgenic technology to develop and improve the traits of this species.

\section{ACKNOWLEDGEMENTS}

This research was supported by a grant from the National Institute of Fisheries Science (NIFS) of the Korea (R2019018).

\section{REFERENCES}

Beardmore JA (1997) Transgenics: Autotransgenics and allotransgenics. Transgenic Res 6:107-108.

Bunnell TM, Burbach BJ, Shimizu Y, Ervasti JM (2011) $\beta$ Actin specifically controls cell growth, migration, and the G-actin pool. Mol Biol Cell 22:4047-4058.

Dominguez R, Holmes KC (2011) Actin structure and function. Annu Rev Biophys 40:169-186.

Goto R, Saito T, Kawakami Y, Kitauchi T, Takagi M, Todo T, Arai K, Yamaha E (2015) Visualization of primordial germ cells in the fertilized pelagic eggs of the barfin flounder Verasper moseri. Int J Dev Biol 59:465470.Gustafson TA, Keds LH (1989) Identification of mutiple proteins that interact with functional regions of the human cardiac alpha-actin promoter. Mol Cell Biol 9: 3269-3283.

Higashijima S, Okamoto H, Ueno N, Hotta Y, Eguchi G (1997) High-frequency generation of transgenic zebrafish which reliably express GFP in whole muscles or the whole body by using promoters of zebrafish origin. Dev Biol 192:289-299.

Kim S, Karsi A, Dunham RA, Liu Z (2000) The skeletal muscle alpha-actin gene of channel catfish (Ictalurus punctatus) and its association with piscine specific SINE elements. Gene 252:173-181.

Kim JE, Lee YM, Lee JH, Noh JK, Kim HC, Park CJ, Park JW, Kim KK (2014) Development and validation of single nucleotide polymorphism (SNP) Markers from an expressed sequence tag (EST) database in olive flounder (Paralichthys olivaceus). Dev Reprod 18:275286.

Kong HJ, Lee YJ, Kim WJ, Kim HS, Kim BS, An CM, Yeo SY, Cho HK (2013) Molecular and functional analyses of the fast skeletal myosin light chain2 gene of the Korean oily bitterling, Acheilognathus koreensis. Int J Mol Sci 14:16672-16684.

Krasnov A, Teerijoki H, Gorodilov Y, Molsa H (2003) Cloning of rainbow trout (Oncorhynchus mykiss) alpha-actin, myosin regulatory light chain genes and the 5'-flanking region of alpha-tropomyosin: Functional assessment of promoters. J Exp Biol 206:601-608.

Kusakabe R, Kusakabe T, Suzuki N (1999) In vivo analysis of two striated muscle actin promoters reveals combinations of multiple regulatory modules required for skeletal and cardiac muscle-specific gene expression. Int J Dev Biol 43:541-554.

Mclean N, Talwar S (1984) Injection of cloned genes into rainbow trout eggs. J Embyol Exp Morpholog 82:187.

Minty A, Kedes L (1986) Upstream regions of the human cardiac actin gene that modulate its transcription in muscle cells: Presence of an evolutionarily conserved repeated motif. Mol Cell Biol 6:2125-2136.

Miwa T, Kedes L (1987) Duplicated CArG box domains have positive and mutually dependent regulatory roles in expression of the human alpha-cardiac actin gene. Mol Cell Biol 7:2803-2813.

Moll R, Holzhausen H, Mennel HD, Kuhn C, Baumann R, Taege C, Franke WW (2006) The cardiac isoform of alpha-actin in regenerating and atrophic skeletal muscle, myopathies and rhabdomyomatous tumors: An immunohistochemical study using monoclonal antibodies. 


\section{Alpha Actin 1 Promoter-Driven RFP Expression in Olive Flounder}

Virchows Arch 449:175-191.

Sartorelli V, Webster KA, Kedes L (1990) Muscle-specific expression of the cardiac alpha-actin gene requires MyoD1, CArG-box binding factor, and Sp1. Genes Dev 4:1811-1822.

Shao C, Bao B, Xie Z, Chen X, Li B, Jia X, Yao Q, Orti G, Li W, Li X, Hamre K, Xu J, Wang L, Chen F, Tian Y, Schreiber AM, Wang N, Wei F, Zhang J, Dong Z, Gao L, Gai J, Sakamoto T, Mo S, Chen W, Shi Q, Li H, Xiu Y, Li Y, Xu W, Shi Z, Zhang G, Power DM, Wang Q, Schartl M, Chen S (2017) The genome and transcriptome of Japanese flounder provide insights into flatfish asymmetry. Nat Genet 49:119-124.

Stanke M, Tzvetkova A, Morgenstern B (2006) AUGUSTUS at EGASP: Using EST, protein and genomic alignments for improved gene prediction in the human genome. Genome Biol 7:S11.

Sakakura Y (2006) Larval fish behavior can be a predictable indicator for the quality of Japanese flounder seedlings for release. Aquaculture 257:316-320.

Tonelli FMP, Lacerda SMSN, Tonelli FCP, Costa GMJ, de Franca LR, Resende RR (2017) Progress and biotechnological prospects in fish transgenesis. Biotechnol Adv 35:832-844.

Venkatesh B, Tay BH, Elgar G, Brenner S (1996) Isolation, characterization and evolution of nine pufferfish (Fugu rubripes) actin genes. J Mol Biol 259:655-665.

Westerfield M (1995) The zebrafish book: a guide for the laboratory use of zebrafish (Danio Rerio). Univ. of Oregon, Eugene, OR. 